\title{
Random attractor of stochastic partly dissipative systems perturbed by Lévy noise
}

\author{
Jianhua Huang
}

Correspondence: jhhuang32@nudt. edu.cn

Department of Mathematics, National University of Defense and Technology, Changsha 410073, China

\begin{abstract}
The current paper is devoted to random dynamics of stochastic partly dissipative systems perturbed by Lévy noise. By the technique of dissipative in probability and multivalued random dynamical systems (MRDS), the existences of random attractor for MRDS generated by the stochastically perturbed partly dissipative systems are provided, both the weaker restrictions and stronger restrictions on the coefficients of Lévy noise respectively.

Mathematics Subject Classification: 35R60; 60H15; 35K57.
\end{abstract}

Keywords: multivalued random dynamical systems, random attractor, dissipative in probability, Lévy noise

\section{Introduction}

Global attractors play an important role in the study of asymptotic behavior of various nonlinear systems. There is a great amount of works toward the global attractors for dissipative autonomous as well as nonautonomous and random equations, see [1-7], etc. Dynamical systems driven by non-Gaussian processes, such as Lévy processes, have attracted a lot of attention recently. Stochastic differential equations driven by Lévy processes have been summarized in [8]. Peszat and Zabczyk [9] have presented a basic framework for partial differential equations driven by Lévy processes, which extended several results known for stochastic partial differential equations (SPDEs) driven by Wiener processes. For more works on SPDEs driven by Lévy processes, see [9] and references therein.

Recently, the authors in [6] developed the new frame of the random attractor for infinite dimensional systems, in which, the solution is not necessarily required to be unique, but the corresponding multivalued random dynamical systems (MRDS) is dissipative with probability one. Kapustyan et al. [10] follows the idea of the one in [6], and study the random attractor of reaction diffusion systems perturbed by càdlàg process.

$$
\left\{\begin{array}{l}
\frac{\partial u(t, x)}{\partial t}=a \Delta u(t, x)-f(u(t, x))+h(x)+g(u(t, x)) \eta(t, \omega), \\
\left.u\right|_{\partial Q}=0 . \quad u_{\mid t=0}=u_{0}(x) .
\end{array}\right.
$$

where $a>0, Q \subset R^{n}$ is a bounded domain with smooth boundary, $f, g \in C(R), h \in L^{2}$ (Q), $f$ and $g$ satisfy some appropriate assumptions, and $\eta(t, \omega)$ is a stochastic càdlag process with right continuity and left limits.

(C) 2012 Huang; licensee Springer. This is an open access article distributed under the terms of the Creative Commons Attribution License (http://creativecommons.org/licenses/by/2.0), which permits unrestricted use, distribution, and reproduction in any medium, provided the original work is properly cited. 
Global attractors for deterministic FitzHugh-Nagumo systems and partly dissipative deterministic reaction diffusion systems have been investigated in [11] for the bounded domain case and in [7] for the unbounded domain case. Regarding the stochastic FitzHugh-Nagumo system as well as the following general partly dissipative random reaction diffusion system

$$
\left\{\begin{array}{l}
\frac{\partial u(t, x)}{\partial t}=\Delta u(t, x)+h\left(\theta_{t} \omega, x, u\right)+f\left(\theta_{t} \omega, x, u, v\right) \\
\frac{\partial v(t, x)}{\partial t}=-\sigma v(t, x)+g\left(\theta_{t} \omega, x, u\right) \\
\left.u\right|_{\partial Q}=0 .
\end{array}\right.
$$

where $D \subset R^{n}$ is a smooth bounded domain, $h: \Omega \times D \times R \rightarrow R, f: \Omega \times D \times R^{2} \rightarrow$ $R$ and $g: \Omega \times D \times R \rightarrow R$ are measurable and satisfy some appropriate hypotheses. The authors in [5] show the existence of random attractor for the general random partly dissipative reaction diffusion equation (1.2), and provide the Hausdorff dimension of random attractor of stochastic FitzHugh-Nagumo systems, [12] shows the existence of pullback attractor of the non-autonomous FitzHugh-Nagumo systems on unbounded domains. The author in [13] study the stochastic partly FitzHugh-Nagumo systems driven by Gaussian white noise, and show the existence of random attractor by uniform estimates on solution for large space and time variable via a cut-off technique.

Motivated by Kapustyan et al. [10], we consider the following partly dissipative reaction diffusion systems perturbed by Lévy noise.

$$
\left\{\begin{array}{l}
\frac{\partial u(t, x)}{\partial t,}-d \Delta u(t, x)+h(x, u)+f(x, u, v)=k_{1}(u) L(t, \omega), \quad(x, t) \in D \times R^{+}, \\
\frac{\partial v(t, x)}{\partial t}+\sigma(x) v+g(x, u)=k_{2}(v) L(t, \omega), \quad(x, t) \in D \times R^{+}, \\
\left.u\right|_{\partial D}=0 . \\
u_{\mid t=0}=u_{0}(x), v_{\mid t=0}=v_{0}(x),
\end{array}\right.
$$

where $d>0, L(t, \omega)$ is a stochastic Lévy process which trajectories are right-continuous and have left limits. Functions $h, f, g, k_{1}, k_{2}$ and $\sigma$ are twice continuously differentiable in all variables and satisfy

(H1) $c_{1}|u|^{p}-c_{3} \leq h(x, u) u \leq c_{2}|u|^{p}+c_{3}, p>2$.

(H2) $|f(x, u, v)| \leq c_{4}\left(1+|u|^{p_{1}}+|v|\right), 0<p_{1}<p-1$.

(H3) $\delta(x) \geq \delta>0$.

(H4) $\left|g^{\prime}(u)\right| \leq c_{5},\left|g_{x_{i}}^{\prime}(x, u)\right| \leq c_{5}(1+|u|), i=1, \ldots, n$, where $\delta_{i}>0, i=1, \ldots, 5$.

(H5) $\left(h_{u}^{\prime}(x, u)+f_{u}^{\prime}(x, u, v)\right) \xi_{1}^{2}+f_{v}^{\prime}(x, u, v) \xi_{1} \xi_{2} \geq-c_{6}\left(\xi_{1}^{2}+\xi_{2}^{2}\right), i=1, \ldots, n$, where $\delta_{6}>0$.

(H6) there exist some positive constants $a_{1}, a_{2}, b_{1}$ and $b_{2}$ such that

$$
\left|k_{1}(u)\right| \leq a_{1}|u|+b_{1}, \quad\left|k_{2}(v)\right| \leq a_{2}|v|+b_{2} .
$$

In current paper, we study the long time behavior of stochastic partly dissipative equations (1.3) perturbed by Lévy noise. By the technique of dissipative in probability and MRDS, the existences of random attractor for MRDS generated by the perturbed partly dissipative systems (1.3) are provided, both the weaker restrictions and stronger restrictions on the coefficients of Lévy noise, respectively.

It is necessary to point out that, comparing with the main result for (1.1) in [10], the two difficulties we need to tackle are the measurability and the asymptotic 
compactness of the solutions operators of stochastic partly dissipative systems (1.3). By the similar arguments in [4], the measurability for stochastic partly dissipative systems perturbed by Lévy noise, so we will use the technique of dissipative in probability and MRDS, to show the existences of random attractor for MRDS generated by the stochastically perturbed partly dissipative systems (1.3).

The rest of the paper is organized as follows. In Section 2, we present some definitions and theorems about MRDS. Section 3 is devoted to the study of the existence of random attractor of partly dissipative random reaction diffusion systems perturbed by Lévy noise in terms of dissipative in probability, the weaker restrictions and stronger restrictions on the coefficients of Lévy noise, respectively.

\section{Multivalued random dynamical systems}

In this section, we recall the definitions of multivalued random dynamical systems and random attractors, the reader is referred to $[6,10]$ for details.

Definition 2.1. [9] Let $E$ be a Banach space, and let $X=(X(t), t \geq 0)$ be a $E$ valued stochastic process defined on a probability space $(\Omega, \mathcal{F}, P)$. It is called as a Lévy process if

(L1) $X(0)=0$, a.s.;

(L2) $X$ has independent and stationary increments; and

(L3) $X$ is stochastically continuous, i.e. for all $\delta>0$ and for all $s \geq 0$

$$
\lim _{t \rightarrow s} P(|X(t)-X(s)|>\delta)=0 .
$$

We can choose the sample space $\Omega=D(R)$ of Lévy process as

$$
\Omega=D(R)=\left\{\omega(\cdot): R \rightarrow R, \quad \forall t \in R, \lim _{s \rightarrow t-} \omega(s)=\omega(t-), \lim _{s \rightarrow t+} \omega(s)=\omega(t)\right\}
$$

It is easy to check that $\Omega=D(R)$ is the Skorokhod metric space with Borel $\sigma$-algebra $\Phi$.

$$
\theta_{t} \omega(\cdot)=\omega(t+\cdot),
$$

$P$ is $\theta_{t}$-invariant probability measure. The matric $\rho$ can be defined by

$$
\rho\left(\omega_{1}, \omega_{2}\right)=\Sigma_{i=1}^{\infty} \frac{1}{2^{i}} \frac{\rho_{i}\left(\omega_{1}, \omega_{2}\right)}{1+\rho_{i}\left(\omega_{1}, \omega_{2}\right)},
$$

where

$$
\rho_{i}\left(\omega_{1}, \omega_{2}\right)=\inf _{\lambda \in \Lambda}\left(\sup _{t \in[-i, i]}\left|\omega_{1}(t)-\omega_{2}(\lambda(t))\right|+\sup _{t \in[-i, i]}|t-\lambda(t)|\right),
$$

$\Lambda=\{\lambda(\cdot) \mid: \lambda(\cdot):[-i, i] \rightarrow[-i, i], \lambda(-i)=-i, \lambda(i)=i, \lambda(\cdot)$ is continuous and monotonically increasing function\}.

Remark 2.1. It follows from [9] that Lévy process $\eta(t, \omega)$ is a stochastic càdlàg process with trajectories without discontinuities of the second kind, that is to say, the sample path is right continuous with left limits.

In this paper, we just use the càdlàg property of the Lévy process, and $L(t, \omega)=\omega(t)=$ $\pi\left(\theta_{t} \omega\right)$, where $\pi: \Omega \rightarrow R$, and $\pi(\omega)=\omega(0)$. 
Let $C(X)$ be the family of all nonempty closed subset of $X, \beta(X)$ be the family of all nonempty and bounded sunset of $X$. In the sequel, we will introduce the definition of MRDS,

Definition 2.2. [10]A multivalued mapping $G: R_{+} \times \Omega \times X \rightarrow C(X)$ is called as a MRDS if

(1) the mapping $(t, x) \rightarrow G(t, \omega) x$ is measurable for all $x \in X$

(2) $G(0, \omega) x=x$ and $G(t+s, \omega) x \subset G\left(t, \theta_{s} \omega\right) G(s, \omega) x$ for all $t, s \in R_{+}, x \in X, \omega \in \Omega$.

Definition 2.3. [10]A measurable set $A(\omega)$ is called a random attractor for a MRDS $G$ if for P-almost all $\omega \in \Omega$,

(1) $A\left(\theta_{t} \omega\right) \subset G(t, \omega) A(\omega)$ for all $t \in R_{+}$;

(2) for all $B \in \beta(X)$, dist $\left(G\left(t, \theta_{-t}(\omega)\right) B, A(\omega)\right) \rightarrow 0, t \rightarrow+\infty$;

(3) $A(\omega)$ is a compact set in $X$.

The following hypotheses is developed by Kapustyan et al. [10], which are key tools to show the existence of random attractor for MRDS generated by stochastic equations (1.3).

(G1) the mapping $(t, \omega) \rightarrow \overline{G(t, \omega) B}$ is measurable for all $B \in \beta(X)$

(G2) for all $\epsilon>0$, there exists $R=R(\epsilon)$ such that for all $B \in \beta(X)$, there exists $T=T$ $(B, R, \epsilon)$ for which

$$
P\left\{\sup _{t \geq T}\left\|G\left(t, \theta_{-t} \omega\right) B\right\|>R\right\}<\varepsilon .
$$

As the authors pointed out in the paper [10] that, the condition (G1) is used to show the measurability of the map, and the condition (G2) is used to show the MRDS is dissipative in probability.

For a given $B \in \mathcal{B}(X)$, define

$$
\wedge_{B_{n}}(\omega)=\bigcap_{T>0} \overline{\bigcup_{t \geq T} G\left(t, \theta_{-t} \omega\right) B}, \quad \mathcal{A}=\overline{\bigcup_{B \in \mathcal{B}(X)} \wedge_{B_{n}}(\omega)}=\overline{\bigcup_{k=1}^{\infty} \wedge_{B_{n}}(\omega)} .
$$

Then, $\wedge_{B_{n}}(\omega)$ consists of the limit of all convergent sequences $\left\{\xi_{n}\right\}$, where $\xi_{n} \in G\left(t_{n}, \theta_{-t_{n}} \omega\right) B$, and $t_{n} \rightarrow \infty$.

Theorem 2.1. ( [10], Theorem 1)

Let a mapping $x \rightarrow G(t, \omega) x$ be upper semicontinuous and compact-valued for all $t \in$ $R_{+}$and $\omega \in \Omega$, let conditions (G1) and (G2) hold for a MRDS G and let the set $G(t, \omega)$ $B_{R}$ be precompact in $X$ for all $\omega \in \Omega, t>0$ and $R>0$. Then,

$$
\mathcal{A}(\omega)=\overline{\bigcup_{n=1}^{\infty} \wedge_{B_{n}}(\omega)}
$$

is a random attractor for $G$. Therefore, the attractor is unique, it is a minimal set among closed attracting sets, and it is a maximal set among compact, measurable, semiinvariant sets. 


\section{Random attractor for MRDS of (1.3)}

In this section, we will prove the solution of equation (1.3) can generate a MRDS, and the MRDS posses a random attractor.

Lemma 3.1. Under the assumptions ( $\mathrm{H} 1)-(\mathrm{H} 5)$, there exists the positive constants $m_{1}$, $m_{2}, \delta_{3}, c_{a}, c_{r}$ such that the solution $((u(t), v(t))$ of the equations (1.3) satisfy the following estimates.

$$
\begin{aligned}
|u(t)|^{2}+|v(t)|^{2} \leq & \left(|u(0)|^{2}+|v(0)|^{2}\right) e^{\left(\int_{0}^{t}\left(m_{1}|\omega(p)|-m_{2}\right) \mathrm{d} p\right)} \\
& +\int_{0}^{t}\left(c_{3}|D|+\delta_{3}+\left(c_{a}+c_{r}\right)|\omega(s)|\right) e^{\left(\int_{s}^{t}\left(m_{1}|\omega(p)|-m_{2}\right) \mathrm{d} p\right)} \mathrm{d} s .
\end{aligned}
$$

Proof. Multiplying the first equation of system (1.3) by $u$ and the second equation of system (1.3) by $v$, Integrating them over $D$, we have

$$
\begin{aligned}
& \frac{1}{2} \frac{\mathrm{d}}{\mathrm{d} t}\left(|u|^{2}+|v|^{2}\right)+d\|u\|^{2}+\int_{D} \sigma(x) v^{2} \mathrm{~d} x+\int_{D} h(x, u) u \mathrm{~d} x+\int_{D}[f(x, u, v) u+g(x, v) v] \mathrm{d} x \\
& =\int_{D} k_{1}(x, u) \omega(t) u \mathrm{~d} x+\int_{D} k_{2}(x, v) \omega(t) v \mathrm{~d} x .
\end{aligned}
$$

It follows from (H6) and Young inequality that there exist two constants $a>0$ and $r>0$ such that

$$
\begin{aligned}
& 2\left(k_{1}(u) \omega(t), u\right) \leq\left(2 a_{1}+r\right)|\omega||u|^{2}+c_{r}|\omega|, \\
& 2\left(k_{2}(v) \omega(t), v\right) \leq\left(2 a_{2}+a\right)|\omega||v|^{2}+c_{a}|\omega| .
\end{aligned}
$$

Due to (H4), there exists a constant $c_{7}>0$ such that

$$
|g(x, \xi)| \leq c_{7}(1+|\xi|), \quad \forall \xi \in R, x \in D
$$

We deduce from (H1) to (H3) that

$$
\begin{aligned}
& \frac{\mathrm{d}}{\mathrm{d} t}\left(|u|^{2}+|v|^{2}\right)+2 d\|u\|^{2}+2 \delta|v|^{2}+2 c_{1} \int_{D}|u|^{\mathrm{p}} \mathrm{d} x \\
& \leq 2 c_{3}|D|+2\left(c_{4}+c_{7}\right) \int_{D}\left(|u|+|u|^{p_{1}+1}\right) \mathrm{d} x+2\left(c_{4}+c_{7}\right) \int_{D}|v|(1+|u|) \mathrm{d} x \\
& +\left(c_{r}+c_{a}\right)|\omega|+\left(2 a_{1}+r\right)|\omega|\|u\|^{2}+\left(2 a_{2}+a\right)|\omega|\|v\|^{2} .
\end{aligned}
$$

Notice that

$$
\left(c_{4}+c_{7}\right) \int_{D}\left(|v|(1+|u|) \mathrm{d} x \leq \frac{\delta}{2} \int_{D}|v|^{2} \mathrm{~d} x+\frac{\left(c_{4}+c_{7}\right)^{2}}{2 \delta} \int_{D}(1+|u|)^{2} \mathrm{~d} x .\right.
$$

Let $q=\max \left\{p_{1}+1,2\right\}$, then there exists a constant $\delta_{2}>0$ such that

$$
\left(c_{4}+c_{7}\right)\left(|\xi|+|\xi|^{p_{1}+1}+\frac{\left(c_{4}+c_{7}\right)^{2}}{2 \delta}\left(1+|\xi|^{2}\right) \leq \delta_{2}\left(|\xi|^{q}+1\right)\right)
$$


Thus,

$$
\left(c_{4}+c_{7}\right) \int_{D}\left(|u|+|u|^{p_{1}+1}+\frac{\left(c_{4}+c_{7}\right)^{2}}{2 \delta}(1+|u|)^{2}\right) \mathrm{d} x \leq \frac{c_{1}}{4} \int_{D}|u|^{p} \mathrm{~d} x+\delta_{3} .
$$

Hence

$$
\begin{aligned}
& \frac{\mathrm{d}}{\mathrm{d} t}\left(|u|^{2}+|v|^{2}\right)+2 d\|u\|^{2}+\delta|v|^{2}+\frac{3}{2} c_{1}|u|_{p}^{p} \\
& \quad \leq c_{3}|D|+\delta_{3}+\left(c_{a}+c_{r}\right)|\omega|+\left(2 a_{1}+r\right)|\omega||u|^{2}+\left(2 a_{2}+a\right)|\omega||v|^{2} .
\end{aligned}
$$

Let $m_{1}=\max \left\{2 a_{1}+r, 2 a_{2}+a\right\}, m_{2}=\min \left\{\delta, 2 d \lambda_{1}\right\}$, where $\lambda_{1}>0$ is the first eigenvalue of $-\Delta$ in $H_{0}^{1}(D)$. By the inequality (3.1), we have

$$
\frac{\mathrm{d}}{\mathrm{d} t}\left(|u|^{2}+|v|^{2}\right) \leq c_{3}|D|+\delta_{3}+\left(c_{a}+c_{r}\right)|\omega|+\left(m_{1}|\omega(t)|-m_{2}\right)\left(|u|^{2}+|v|^{2}\right) .
$$

Integrating (3.2) on the interval $[s, t](t \geq s \geq 0)$, we obtain that

$$
\begin{aligned}
|u(t)|^{2}+|v(t)|^{2} \leq & |u(s)|^{2}+|v(s)|^{2}+\left(c_{3}|D|+\delta_{3}\right)(t-s)+\left(c_{a}+c_{r}\right) \int_{s}^{t}|\omega(p)| \mathrm{d} p \\
& +\int_{s}^{t}\left(m_{1}|\omega(p)|-m_{2}\right)\left(|u(p)|^{2}+|v(p)|^{2}\right) \mathrm{d} p .
\end{aligned}
$$

Using the Gronwall's Lemma,

$$
\begin{aligned}
|u(t)|^{2}+|v(t)|^{2} \leq & \left(|u(0)|^{2}+|v(0)|^{2}\right) e^{\left(\int_{0}^{t}\left(m_{1}|\omega(p)|-m_{2}\right) \mathrm{d} p\right)} \\
& +\int_{0}^{t}\left(c_{3}|D|+\delta_{3}+\left(c_{a}+c_{r}\right)|\omega(s)|\right) e^{\left(\int_{s}^{t}\left(m_{1}|\omega(p)|-m_{2}\right) \mathrm{d} p\right)} \mathrm{d} s .
\end{aligned}
$$

for all $t>0$. The proof of Lemma 3.1 is completed.

Lemma

3.2 .

Let

$\left\{\left(u_{n}, v_{n}\right)=\left(u_{n}\left(t, \omega_{n}\right)\left(u_{n}^{0}, v_{n}^{0}\right), v_{n}\left(t, \omega_{n}\right)\left(u_{n}^{0}, v_{n}^{0}\right),\right)\right\} \subset\left(L^{2}\left(0, T ; H_{0}^{1}(D)\right) \bigcap L^{\mathcal{P}}\left(D \times(0, T) \bigcap C\left(0, \infty ; L^{2}(D)\right) \times C\left(0, \infty ; L^{2}(D)\right)\right.\right.$ be an arbitrary sequence of solution of equation (1.1), where $\omega_{n} \rightarrow \omega_{0}$ in $\Omega, u_{n}^{0} \rightarrow u_{0}$ weakly in $L^{2}$ $\times L^{2}$, and $t_{n} \rightarrow t_{0}>0$. Then,

$$
\left(u_{n}\left(t_{n}, \omega_{n}\right), v_{n}\left(t_{n}, \omega_{n}\right)\left(u_{n}^{0}, v_{n}^{0}\right) \rightarrow\left(u\left(t_{0}, \omega_{0}\right), v\left(t_{0}, \omega_{0}\right)\left(u_{0}, v_{0}\right) .\right.\right.
$$

in $L^{2} \times L^{2}$ at least along some subsequence where $(u, v)=\left(u\left(t, \omega_{0}\right), v\left(t, \omega_{0}\right)\left(u_{0}, v_{0}\right) \in\left(L^{2}\left(0, T ; H_{0}^{1}(D)\right) \bigcap L^{p}\left(D \times(0, T) \bigcap C\left(0, \infty ; L^{2}(D)\right) \times C\left(0, \infty ; L^{2}(D)\right)\right.\right.\right.$ is the solution of equation (1.3).

Proof. Let $T>0$. It follows from (3.1) that the sequence $\left\{\left(\left(u_{n}, v_{n}\right)\right)\right\}$ is bounded in $\left(L^{p}\left(0, T, L^{p}(D)\right) \bigcap L^{2}\left(0, T, H_{0}^{1}(D)\right) \cap L^{\infty}\left(0, T, L^{2}(D)\right)\right) \times C\left(0, T, L^{2}(D)\right)$. By the similar argument of Proposition 1.1 that there exists a subsequence $\left.u_{n}\left(\cdot, \omega_{n}\right)\left(u_{n^{\prime}}^{0}, v_{n}^{0}\right)\right) \rightarrow(u(\cdot)$, $v(\cdot))$ in $L^{2}\left(0, T, L^{2}(D)\right) \times L^{2}\left(0, T, L^{2}(D)\right),\left(u_{n}\left(t, \omega_{n}\right), v_{n}\left(t, \omega_{n}\right)\right)\left(u_{n}^{0}, v_{n}^{0}\right) \rightarrow(u(t), v(t))$ strongly in $\left.L^{2}(D) \times L^{2}(D)\right)$ for almost all $t \in(0, T)$ and weakly in $\left.L^{2}(D) \times L^{2}(D)\right)$ uniformly in $t \in[0, T]$. 
Let

$$
\begin{gathered}
L_{n}(p)=\max \left\{2 a_{1}+r, 2 a_{2}+\alpha\right\}\left|\omega_{n}(p)\right|-\min \left\{\delta, 2 d \lambda_{1}\right\}=m_{1}\left|\omega_{n}(p)\right|-m_{2} \\
L_{0}(p)=\max \left\{2 a_{1}+r, 2 a_{2}+\alpha\right\}\left|\omega_{0}(p)\right|-\min \left\{\delta, 2 d \lambda_{1}\right\}=m_{1}\left|\omega_{0}(p)\right|-m_{2}
\end{gathered}
$$

and denote

$$
\begin{aligned}
J_{n}\left(t, \omega_{n}\right)= & \left(\left|u_{n}(t)\right|^{2}+\left|v_{n}(t)\right|^{2}\right)-\left(c_{3}|D|+\delta_{3}\right) t-\left(c_{\alpha}+c_{\gamma}\right) \int_{0}^{t}\left|\omega_{n}(p)\right| \mathrm{d} p \\
& -\int_{0}^{t} L_{n}(p)\left(\left|u_{n}(p)\right|^{2}+\left|v_{n}(p)\right|^{2}\right) \mathrm{d} p, \\
J_{0}\left(t, \omega_{0}\right)= & \left(|u(t)|^{2}+|v(t)|^{2}\right)-\left(c_{3}|D|+\delta_{3}\right) t-\left(c_{\alpha}+c_{\gamma}\right) \int_{0}^{t}\left|\omega_{0}(p)\right| \mathrm{d} p \\
& -\int_{0}^{t} L_{0}(p)\left(|u(p)|^{2}+|v(p)|^{2}\right) \mathrm{d} p .
\end{aligned}
$$

It follows from (3.3) that

$$
J\left(t, \omega_{0}\right) \leq J\left(s, \omega_{0}\right), \quad J_{n}\left(t, \omega_{n}\right) \leq J_{n}\left(s, \omega_{n}\right)
$$

for all $t \geq s, t, s \in[0, T]$, and $J_{n}(t, \omega) \rightarrow J\left(t, \omega_{0}\right)$ for almost all $t \in(0, T)$. It is easy to check that $J_{n}\left(t, \omega_{n}\right) \rightarrow J\left(t, \omega_{0}\right)$ uniformly on an arbitrary interval $[a, b] \subset(0, T)$. The convergence $\omega_{n} \rightarrow \omega_{0}$ implies that $L_{n}(\cdot) \rightarrow L_{0}(\cdot)$ in $L^{1}(0, t)$ and $L_{n}(\cdot), n \geq 1$ are bounded in $L^{\infty}(0, t)$. Then

$$
\int_{0}^{t} L_{n}(p)\left(\left|u_{n}(p)\right|^{2}+\left|v_{n}(p)\right|^{2}\right) \mathrm{d} p \rightarrow \int_{0}^{t} L_{0}(p)\left(|u(p)|^{2}+\left|v_{n}(p)\right|^{2}\right) \mathrm{d} p .
$$

Hence,

$$
\begin{aligned}
& \liminf _{n \rightarrow \infty} J_{n}\left(t_{n}, \omega_{n}\right)=J\left(t_{0}, \omega_{0}\right) \\
& \geq \liminf _{n \rightarrow \infty}\left(\left|u_{n}\right|^{2}+\left|v_{n}\right|^{2}\right)-\left(c_{3}|D|+\delta_{3}\right) t_{0}-\left(c_{\alpha}+c_{\gamma}\right) \int_{0}^{t_{0}}\left|\omega_{0}(p)\right| \mathrm{d} p \\
& -\int_{0}^{t_{0}} L_{0}(p)\left(|u(p)|^{2}+|v(p)|^{2}\right) \mathrm{d} p
\end{aligned}
$$

for all $t_{n} \geq t_{0}>0$.

Hence

$$
\left|u\left(t_{0}\right)\right|+\left|v\left(t_{0}\right)\right| \geq \liminf _{n \rightarrow \infty}\left(\left|u_{n}\left(t_{n}\right)\right|+\left|v_{n}\left(t_{n}\right)\right|\right) .
$$

Since $\left(u_{n}\left(t_{n}\right), v_{n}\left(t_{n}\right)\right) \rightarrow\left(u\left(t_{0}\right), v\left(t_{0}\right)\right)$ weakly in $L^{2}(D) \times L^{2}(D)$. The converse inequality also holds and therefore

$$
\liminf _{n \rightarrow \infty}\left(\left|u_{n}\left(t_{n}\right)\right|+\left|v_{n}\left(t_{n}\right)\right|\right) \leq\left(\left|u\left(t_{0}\right)\right|+\left|v\left(t_{0}\right)\right|\right) \leq \liminf _{n \rightarrow \infty}\left(\left|u_{n}\left(t_{n}\right)\right|+\left|v_{n}\left(t_{n}\right)\right|\right) .
$$


Moreover, $\left(u_{n}\left(t_{n}\right), v_{n}\left(t_{n}\right)\right)$ converges strongly to $\left(u\left(t_{0}\right), v\left(t_{0}\right)\right)$ in $L^{2}(D) \times L^{2}(D)$. The proof of Lemma 3.2 is completed.

Lemma 3.3. Let $\Omega$ be a metric space and $\Phi$ be a Borel $\sigma$-algebra. Assume that a multivalued mapping $G: R_{+} \times \Omega \times X \rightarrow C(X)$ satisfies the following condition: if $x_{n} \rightarrow$ $x$ weakly in $X$ as $t_{n} \rightarrow t_{0}>0, \omega_{n} \rightarrow \omega$ in $\Omega$ and $y_{n} \in G\left(t_{n}, \omega_{n}\right) x_{n}$ then $y_{n} \rightarrow y_{0} \in G\left(t_{0}\right.$, $\left.\omega_{0}\right) x_{0}$ in $X$ for some subsequence. Then, assumption $G_{1}$ holds for the mapping $G$.

Proof. The proof is similar to the argument of Lemma 4 in [10], and is omitted here.

Lemma 3.4. For $\left(u_{0}, v_{0}\right) \in L^{2}(D) \times L^{2}(D)$, there exists an unique solution $(u, v) \in C$ $\left(0, \quad \infty ; \quad L^{2}(D) \times L^{2}(D)\right)$ of the equations (1.3) satisfying $u \in L^{2}\left(0, T ; H_{0}^{1}(D)\right) \bigcap L^{p}(D \times(0, T))$. The mapping defined by

$$
G(t, \omega):\left(u_{0}, v_{0}\right) \rightarrow(u(t), v(t))
$$

is a multivalued random dynamical system generated by equation (1.3).

Proof. By the same proof as that of Property 1.1 in [11] and the classical Galerkin approximates, noting that $k_{1}(u) \omega(t)$ and $k_{2}(v) \omega(t)$ are right continuous with left limit with respect to $t$, we can show that the equation (1.3) admits at least a solution $(u(t$, $\left.\left.\omega, u_{0}, v_{0}\right), v\left(t, \omega, u_{0}, v_{0}\right)\right) \in C\left(0, \infty ; L^{2}(D) \times L^{2}(D)\right)$ for every $\omega \in \Omega$. Moreover, $u \in L^{2}\left(0, T ; H_{0}^{1}(D)\right) \bigcap L^{p}(D \times(0, T))$. Similar to the Property 4 in [6], it can be verified the family of mappings $\left\{G(t, \omega)_{t \geq 0}\right.$ generates a MRDS.

Lemma 3.5. Assume that (H1)-(H6) hold, and Levy noise $\omega(t)$ satisfies the following conditions

(HY0) If for all $\epsilon>0, \alpha>0$, there exists $T=T(\epsilon)>0$ such that Lévy noise $\omega(t)$ satisfies

$$
P\left(\omega(t): \sup _{t \geq T} \frac{1}{t} \int_{-t}^{0}|\omega(p)| \mathrm{d} p-\frac{m_{2}}{m_{1}} \leq-\alpha\right)>1-\varepsilon,
$$

(HY1) If for all $\epsilon>0$, there exists a positive constant $D>0$ such that Lévy noise $\omega(t)$ satisfies

$$
\sup _{t \geq 0} P\left\{\omega(t): \int_{-t}^{0}|\omega(s)| e^{m_{1} \alpha s} \mathrm{~d} s \leq D\right\}>1-\varepsilon .
$$

Then, the MRDS G generated by equation (1.3) satisfies the condition $G_{2}$.

Proof. It follows from Lemma 3.1 that there exist random variable $t(\omega) \in\left[T_{1}, T_{2}\right]$ and initial value $x_{0}(\omega) \in B_{r}$ such that the solution of equation (1.3) reaches the superium at $t(\omega)$ for all $T_{2}>T_{1}>0$ and any $\omega \in \Omega$, that is,

$$
\sup _{t \in\left[T_{1}, T_{2}\right]}\left\|G\left(t, \theta_{-t} \omega\right) B_{r}\right\|=\|\left(u\left(t(\omega), \theta_{-t(\omega))} \omega\right), v\left(t(\omega), \theta_{-t(w))} \omega\right)\right)\left(u_{0}(\omega), v_{0}(\omega) \| .\right.
$$

Notes that $\omega \rightarrow \sup _{t \in\left[T_{1}, T_{2}\right]}\left\|G\left(t, \theta_{-t} \omega\right) B_{r}\right\|$ is $\Phi$-measurable, therefore, $\left(u\left(t(\omega), \theta_{-t}\right.\right.$ $(\omega) \omega) x_{0}(\omega)$, and $\left.v\left(t(\omega), \theta_{-t(\omega))} \omega\right)\right)$ are also $\Phi$-measurable.

Fixed $\epsilon>0$, then for any arbitrary $N>0, T$ and for $t \in[T, T+N]$, denote

$$
\begin{aligned}
L^{2} & =\left\{\omega,: \sup \left\|G\left(t, \theta_{-t} \omega\right) B_{r}\right\|^{2}>R^{2}\right\} \\
& =\left\{\omega:\left\|\left(u\left(t(\omega), \theta_{-t(\omega)} \omega\right) x_{0}(\omega), v\left(t(\omega), \theta_{-t(\omega))} \omega\right)\right)\right\|^{2}>R^{2}\right\} .
\end{aligned}
$$


Define

$$
\begin{aligned}
A_{1} & =\left\{\omega: r^{2} e^{m_{1} t(\omega)\left(\frac{1}{t(\omega)} \int_{-t(\omega)}^{0}|\omega(p)| \mathrm{d} p-\frac{m_{2}}{m_{1}}\right)} \geq 1\right\} \\
& \subset\left\{w:\left(\frac{1}{t(\omega)} \int_{-t(\omega)}^{0}|\omega(p)| \mathrm{d} p-\frac{m_{2}}{m_{1}}\right) \geq \frac{1}{m_{1} t(\omega)} \ln \left(\frac{1}{r^{2}}\right)\right\} .
\end{aligned}
$$

Choosing $T=T(r)$ such that

$$
\frac{1}{m_{1} t(\omega)} \ln \left(\frac{1}{r^{2}}\right)>-\alpha, \quad(\alpha>0)
$$

Then,

$$
\begin{aligned}
A_{1} & \subset\left\{\frac{1}{t(\omega)} \int_{-t(\omega)}^{0}|\omega(p)| \mathrm{d} p-\frac{m_{2}}{m_{1}}>-\alpha\right\} \\
& \subset\left\{\omega: \sup _{t \geq T} \frac{1}{t} \int_{-t}^{0}|\omega(p)| \mathrm{d} p-\frac{m_{2}}{m_{1}}>-\alpha\right\} .
\end{aligned}
$$

It follows from (HY0) that there exists a positive constant random variant $T_{1}=T_{1}(\omega)$ such that for $t \geq T_{1}(\epsilon)$,

$$
P\left(\omega \sup _{t \geq T_{1}} \frac{1}{t} \int_{-t}^{0}|\omega(p)| \mathrm{d} p-\frac{m_{2}}{m_{1}} \leq-\alpha\right)>1-\varepsilon
$$

Thus, there exists a random set $A_{2} \subset \Omega$ such that $P\left(A_{2}\right) \leq \frac{\varepsilon}{4}$, and

$$
\sup _{t \geq T_{1}} \frac{1}{t} \int_{-t}^{0}|\omega(p)| \mathrm{d} p-\frac{m_{2}}{m_{1}} \leq-\alpha
$$

for all $\omega \in \Omega \backslash A_{2}$.

Thus, there exists $T_{2}=T_{2}(\epsilon, r) \geq T_{1}+T(r)$ such that

$$
P\left(A_{1}\right)<\frac{\varepsilon}{4}
$$

for all $t(\omega) \in\left[T_{2}, T_{2}+N\right]$.

It follows from (3.1) that

$$
\begin{aligned}
L^{N} \subset & \left\{\omega: r^{2} e^{m_{1} t(\omega)}\left(\frac{1}{t(\omega)} \int_{-t(\omega)}^{0} \mid \omega(p) \mathrm{d} p-\frac{m_{2}}{m_{1}}\right.\right. \\
& +\int_{-t(\omega)}^{0} e^{m_{1} s\left(\int_{s}^{0} \frac{1}{s} \omega(p) \mathrm{d} p+\frac{m_{2}}{m_{1}}\right)}\left(\left(c_{3}|D|+\delta_{3}+\left(c_{r}+c_{a}\right)|\omega(s)|\right) d s>R^{2}\right\} .
\end{aligned}
$$


The definitions $A_{1}$ and $A_{2}$ imply that

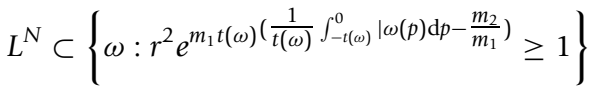

$$
\begin{aligned}
& \bigcup\left\{\omega: \int_{-t(\omega)}^{0} e^{m_{1} s\left(\frac{1}{s} \int_{s}^{0}|\omega(p)| \mathrm{d} p+\frac{m_{2}}{m_{1}}\right)}\left(\left(c_{3}|D|+\delta_{3}+\left(c_{r}+c_{a}\right)|\omega(s)|\right) \mathrm{d} s>R^{2}-1\right)\right\} \\
& \subset A_{1} \bigcup A_{2} \bigcup\left\{\omega: \int_{-T_{1}}^{0}\left(\left(c_{3}|D|+\delta_{3}+\left(c_{r}+c_{a}\right)|\omega(s)|\right) e^{\left\{m_{1} s\left(\frac{1}{s} \int_{s}^{0}|\omega(p)| \mathrm{d} p+\frac{m_{2}}{m_{1}}\right)\right\}} \mathrm{d} s\right.\right. \\
& +\int_{-T_{2}-N}^{0}\left(\left(c_{3}|D|+\delta_{3}+\left(c_{r}+c_{a}\right)|\omega(s)|\right) e^{\alpha m_{1} s} \mathrm{~d} s>R^{2}-1\right\} \\
& =A_{1} \bigcup A_{2} \bigcup\left\{\omega: f_{\varepsilon}(\omega)+\int_{-T_{2}+N}^{0} \mid \omega(s) e^{m_{1} \alpha s} \mathrm{~d} s>\frac{R^{2}-A}{B}\right\} \text {. }
\end{aligned}
$$

where $A$ and $B$ are some positive constants, and $f_{\epsilon}: \omega \rightarrow R$ is a measurable and $P$ -almost everywhere bounded function. Hence there exists a real number $R_{1}=R_{1}(\epsilon)$ and a random set $A_{3} \subset \Omega$ such that

$$
f_{\varepsilon}(\omega)>R_{1}, \quad P\left(A_{3}\right)<\frac{\varepsilon}{4}
$$

for all $\omega \in A_{3}$. Therefore,

$$
L^{N} \subset A_{1} \bigcup A_{2} \bigcup A_{3} \bigcup\left\{\omega: \int_{-T-N}^{0}|\omega(s)| e^{m_{1} \alpha s} \mathrm{~d} s>\frac{R^{2}-A}{B}-R_{1}(\varepsilon)\right\} .
$$

It follows from (HY1) that there exists: a positive constant $D=D(\epsilon)$ such that

$$
P\left\{\omega: \int_{-t}^{0}|\omega(s)| e^{m_{1} \alpha s} d s>D\right\}<\frac{\varepsilon}{4} .
$$

for all $t>0$. Choose $R=R(\epsilon)$ such that

$$
\frac{R^{2}-A}{B}-R_{1}(\varepsilon)>D
$$

Then, for all $\epsilon>0$, there exists $R=R(\epsilon)>0$ for which, whatever $B_{r}$ is a ball with radius $r$, there exists $T=T(\epsilon, R, r)$ such that

$$
P\left(L^{N}\right)=P\left\{\omega: \sup _{t \in[T, T+N]}\left\|G\left(t, \theta_{-t} \omega\right) B_{r}\right\|^{2}>R^{2}\right\}<\varepsilon, \forall N \geq 1 .
$$

Notice that $L^{N} \subset L^{N+1}$, and let $L=\bigcup_{i=1}^{N} L^{N}$, then $P(L)<\epsilon$, and

$$
\left\{\omega: \sup _{t \geq T} \| G\left(t, \theta_{-t} \omega B_{r} \|^{2}>R^{2}\right\}=L,\right.
$$

which implies that G2 holds. Thus, the proof of Lemma (3.5) is completed. 
Theorem 3.1. Assume that conditions (H1)-(H6) hold, and the Lévy noise satisfy the (HYO)- (HY1). Then, the MRDS G generated by the systems (1.3) admits a random attractors.

Proof. It follows from Lemma 3.4 that the equations (1.3) generates a MRDS G. Lemma 3.3 and 3.5 imply that the MRDS $G$ satisfies the conditions $G_{1}$ and $G_{2}$. Then, the existence random attractor is established by theorem (2.1). Thus, the proof is completed.

In the sequel, we are going to show the existence of random attractor for the equation (1.3) with weaker restrictions on the functions $k_{1}(u)$ and $k_{2}(v)$ instead of the stronger restrictions imposed on it, that is to say, $k_{1}(u)$ and $k_{2}(v)$ are assumed to be the functions

$$
\left|k_{1}(u)\right| \leq a_{3}|u|^{\gamma}+b_{3}, \quad\left|k_{2}(v)\right| \leq a_{2}|v|+b_{2} .
$$

for $\gamma<p-1$.

Lemma 3.6. Assume that (H1)-(H5) and (3.6) hold. Then there exists the some positive constants $m_{2}, a_{5}$ and $b_{5}$ such that the solution $(u(t), v(t))$ of the equations (1.3) satisfy the following estimates

$$
\begin{aligned}
|u(t)|^{2}+|v(t)|^{2} \leq & \left(|u(0)|^{2}+|v(0)|^{2}\right) e^{\left.\int_{0}^{t}\left(2 a_{2}+\alpha\right)|\omega(p)|-m_{2}\right) \mathrm{d} p} \\
& +\int_{0}^{t}\left(b_{5}+a_{5}|\omega(s)|^{\frac{p}{p-r-1}}\right) e^{\left.\int_{0}^{t}\left(2 a_{2}+\alpha\right)|\omega(p)|-m_{2}\right) \mathrm{d} p} \mathrm{~d} s .
\end{aligned}
$$

Proof. By the Young inequality and (3.6), we have

$$
2\left(k_{1}(u) \omega(t), u\right) \leq 2 a_{3}|\omega| \cdot\|u\|_{L^{r+1}}^{r+1}+2 b_{3}|\omega| \int_{D}|u| d x \leq a_{4}|\omega|^{\frac{p}{p-r-1}}+\frac{3}{2} c_{1}\|u\|_{L^{p}}^{p}+b_{4} .
$$

Multiplying the first equation of (1.3) by $u$ and the second one by $v$, and integrate over $D$, we obtain

$$
\begin{aligned}
& \frac{\mathrm{d}}{\mathrm{d} t}\left(|u|^{2}+|v|^{2}\right)+2 d\|u\|^{2}+\delta|v|^{2} \\
& \leq c_{3}|D|+\delta_{3}+b_{4}+a_{4}|\omega|^{\frac{p}{p-r-1}}+\left(2 a_{2}+\alpha\right)|\omega| \cdot|v|^{2}+c_{\alpha}|\omega| \\
& \leq b_{5}+a_{5}|\omega|^{\frac{p}{p-r-1}}+\left(2 a_{2}+\alpha\right)|\omega| \cdot|v|^{2} \\
& \leq b_{5}+a_{5}|\omega|^{\frac{p}{p-r-1}}+\left(2 a_{2}+\alpha\right)|\omega| \cdot\left(|u|^{2}+|v|^{2}\right) .
\end{aligned}
$$

Let $m_{2}=\min \left\{\delta, 2 d \lambda_{1}\right\}$, where $\lambda_{1}>0$ is the first eigenvalue of $-\Delta$ in $H_{0}^{1}(D)$. Then

$$
\frac{\mathrm{d}}{\mathrm{d} t}\left(|u|^{2}+|v|^{2}\right) \leq b_{5}+a_{5}|\omega|^{\frac{p}{p-r-1}}+\left(\left(2 a_{2}+\alpha\right)|\omega|-m_{2}\right) \cdot\left(|u|^{2}+|v|^{2}\right) .
$$

Integrating (3.7) on the interval $[s, t](t \geq s \geq 0)$, we get

$$
\begin{aligned}
|u(t)|^{2}+|v(t)|^{2} \leq & |u(s)|^{2}+|v(s)|^{2}+b_{5}(t-s)+a_{5} \int_{s}^{t}|\omega(s)|^{\frac{p}{p-r-1}} \mathrm{~d} s \\
& \left.+\int_{s}^{t}\left(\left(2 a_{2}+\alpha\right)|\omega(p)|-m_{2}\right) \cdot\left(|u(p)|^{2}+|v(p)|^{2}\right) \mathrm{d} p\right) .
\end{aligned}
$$


Applying the Gronwall's Lemma to (3.8) gives

$$
\begin{aligned}
|u(t)|^{2}+|v(t)|^{2} \leq & \left(|u(0)|^{2}+|v(0)|^{2}\right) e^{\left.\int_{0}^{t}\left(2 a_{2}+\alpha\right)|\omega(p)|-m_{2}\right) \mathrm{d} p} \\
& +\int_{0}^{t}\left(b_{5}+a_{5}|\omega(s)|^{\frac{p}{p-r-1}}\right) e^{\left.\int_{0}^{t}\left(2 a_{2}+\alpha\right)|\omega(p)|-m_{2}\right) \mathrm{d} p} \mathrm{~d} s .
\end{aligned}
$$

for all $t>0$. Thus, we complete the proof of Lemma 3.6.

Lemma 3.7. Assume that (H1)-(H5) and (3.6) hold, and Lévy noise $\omega(t)$ satisfies the following conditions

(HY2) If for all $\epsilon>0, \alpha>0$, there exists $T=T(\epsilon)>0$ such that Levy noise $\omega(t)$ satisfies

$$
P\left(\left(\omega(t): \sup _{t \geq T} \frac{1}{t} \int_{-t}^{0}|\omega(p)| \mathrm{d} p-\frac{m_{2}}{2 a_{2}+\alpha} \leq-\gamma\right)>1-\varepsilon,\right.
$$

(HY3) If for all $\epsilon>0$, there exists a positive constant $D>0$ such that Levy noise $\omega(t)$ satisfies

$$
\sup _{t \geq 0} P\left\{\omega(t): \int_{-t}^{0}\left(a_{5}|\omega(s)|^{\frac{p}{p-r-1}}+b_{5}\right) e^{\beta\left(2 a_{2}+\alpha\right) s} \leq D\right\}>1-\varepsilon .
$$

Then, the MRDS G generated by equation (1.3) satisfies the condition $G_{2}$.

Proof. The proof is similar to the one of Lemma 3.5, and is omitted here.

Theorem 3.2. Assume that conditions (H1)-(H5) and (3.6) hold, and the Lévy noise satisfy the (HY2)-(HY3). Then, the MRDS G generated by the systems (1.3) admits a random attractors.

Proof. The proof is similar to the one of Theorem 3.1, and is omitted here.

Remark 3.1. From the proof of Theorem 3.1 and Theorem 3.2, the Theorem 3.1 and Theorem 3.2 hold for more general càdlàg process.

Acknowledgements

This article was supported by the NSF of China (No. 10971225) NSF of Hunan (No. 11JJ3004) and sponsored by SRF for ROCS, SEM, China.

\section{Competing interests}

The authors declare that they have no competing interests.

Received: 24 February 2012 Accepted: 11 June 2012 Published: 11 June 2012

\section{References}

1. Chepyzhov, V, Vishik, M: Attractors for Equations of Mathematical Physics. American Mathematical Society, Providence, Rhode Island (2002)

2. Crauel, H, Flandoli, F: Attractor for random dynamical systems. Probab Theory Relat Fields. 100, 365-393 (1994). doi:10.1007/BF01193705

3. Crauel, H, Debussche, A, Flandoli, F: Random attractors. J Dyn Differ Equ. 9, 307-341 (1997). doi:10.1007/BF02219225

4. Huang, J, Shen, W: Pullback attractors for nonautonomous and random parabolic equations on non-Smooth domains. Discret Contin Dyn Syst. 24, 855-882 (2009)

5. Huang, J, Shen, W: Global sttractors for partly dissipative random/stochastic reaction diffusion equations. Int J Evol Equ. 4, 383-412 (2010)

6. Caraballo, T, Langa, JA, Valero, J: Global attractors for multivalued random dynamical systems. Nolinear Anal. 48 805-829 (2002). doi:10.1016/S0362-546X(00)00216-9

7. Rodriguez-Bernal, A, Wang, B: Attractor for partly dissipative reaction diffusion system in $R^{n}$. J Math Anal Appl. 252, 790-803 (2000). doi:10.1006/jmaa.2000.7122

8. Applebaum, D: Levy Processes and Stochastic Calculus. Cambridge University Press, Cambridge, 2 (2009) 
9. Peszat, S, Zabczyk, J: Stochastic Partial Differential Equations with Levy noise-Evolution Equation Approach. Cambridge University Press, Cambridge (2009)

10. Kapustyan, OV, Valero, J, Pereguda, OV: Random attractor for the reaction diffusion equation pertubed by a stochastic Cadag process. Theor Probab Math Stat. 73, 57-69 (2006)

11. Marion, M: Finite-dimensional attractor associated with partly dissipative reaction diffusion equations. SIAM J Math Anal. 20, 816-844 (1989). doi:10.1137/0520057

12. Wang, B: Pullback attractor for the non-autonomous FitzHugh-Nagumo systems on un-bounded domains. Nonlinear Anal. 70, 3799-3815 (2009). doi:10.1016/j.na.2008.07.011

13. Wang, B: Random attractor for stochastic FitzHugh-Nagumo systems on unbounded domains. Nonlinear Anal. 71 2811-2828 (2009). doi:10.1016/j.na.2009.01.131

doi:10.1186/1029-242X-2012-132

Cite this article as: Huang: Random attractor of stochastic partly dissipative systems perturbed by Lévy noise. Journal of Inequalities and Applications 2012 2012:132.

\section{Submit your manuscript to a SpringerOpen ${ }^{\circ}$} journal and benefit from:

- Convenient online submission

- Rigorous peer review

- Immediate publication on acceptance

- Open access: articles freely available online

- High visibility within the field

- Retaining the copyright to your article

Submit your next manuscript at $\gg$ springeropen.com 